
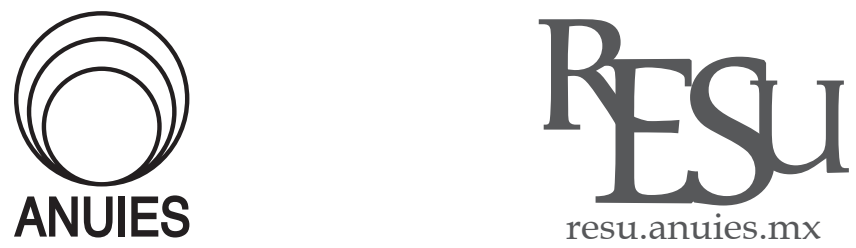

ARTí́CULO

\title{
La evaluación en educación superior con fines de acreditación de alta calidad a través de un modelo sistémico con teoría de redes
}

\author{
Assessment in higher education for high quality accreditation purpose \\ through a systemic model with network theory
}

\section{Frederick Andrés Mendoza Lozano, Mary Natalia Ortegon Cifuentes.}

Institución Universitaria Politécnico Grancolombiano

Correo electrónico: famendozal23@gmail.com

Recibido el 29 de diciembre del 2018; aceptado el 29 de octubre del 2019

\section{Resumen}

Reconociendo algunas falencias identificadas en estudios previos sobre los retos del sistema de aseguramiento de la calidad colombiano, se propone un método para ponderar las características del modelo de acreditación de alta calidad. Este proceso es la base de un abordaje de complejidad en la calidad, que le da nuevas posibilidades de interpretación a todo el ejercicio de autoevaluación y mejora continuas. La metodología es una aplicación innovadora de la teoría de redes. Después de un piloto de aplicación, se concluye que la ponderación obtenida es además una herramienta de gestión replicable en otras instituciones $\mathrm{u}$ otros modelos de acreditación

Palabras clave: Acreditación; Educación superior; Calidad, Teoría de redes; Complejidad 


\begin{abstract}
After recognizing some shortcomings identified in previous studies of the Colombian quality assurance system challenges, a method is proposed to weigh the characteristics of the high-quality accreditation model. This process is the basis of a complexity approach to quality, which gives new possibilities of interpretation to the entire exercise of selfassessment and continuous improvement. The methodology is an innovative application of network theory. After an application pilot, it is concluded that the weighting obtained is also a replicable management tool in other institutions or other accreditation models.
\end{abstract}

Keywords: Accreditation; Higher education; Quality; Network theory; Complexity

\title{
Introducción
}

$\mathrm{E}$ proceso de acreditación colombiano ha ido ganando legitimidad en las Instituciones de Educación Superior (IES). Las IES más prestigiosas obtienen ese reconocimiento en la mayoría de sus programas y a nivel institucional. Sin embargo, en general para todo el sistema, el camino no ha sido fácil: según el Sistema Nacional de Información de la Educación Superior (SNIES), a segundo semestre de 2018 en Colombia, había 13.598 programas de educación superior en todos los niveles; de éstos, 3.682 son del nivel de especialización, que por ley no son acreditables. Del total de programas diferentes al nivel de especialización, 1.399 tienen reconocimiento de acreditación alta calidad, es decir, cerca de un 10\%. Este escenario es preocupante teniendo en cuenta que el Sistema Nacional de Acreditación (SNA) se creó con la ley 30 de 1992, que reglamenta la Educación Superior en Colombia. Por lo tanto, ya casi se completa la tercera década y aún no se logra certificar, en niveles de excelencia, un número significativo de programas. Más aun, según cifras oficiales del Ministerio Educación Nacional (MEN), el número total de procesos con acreditación negada ha aumentado; y ha disminuido el número de acreditaciones concedidas (A 2016, 77 acreditaciones negadas y 56 concedidas) (Colombia. Ministerio de Educación Nacional, 2018).

Este trabajo tiene el propósito de hacer algunos aportes para mejorar el comportamiento de esas cifras y la calidad de la educación superior a través de innovaciones metodológicas en los procesos de autoevaluación con fines de acreditación. Para eso se señalarán tres problemas fundamentales de los procesos de autoevaluación, identificados con base en la experiencia de los 
autores y en estudios previos sobre el caso colombiano (Blasco, 2011; Colombia. Ministerio de Educación Nacional, 2018; Gómez Campo \& Celis Giraldo, 2009), para señalar luego que las necesarias innovaciones metodológicas se hacen viables al reconocer el carácter complejo del concepto de calidad y en consecuencia el uso de nuevas herramientas para caracterizarla y medirla. En particular se ilustrarán las ventajas de usar la teoría de redes como aporte al problema de la ponderación y el descubrimiento de propiedades complejas.

\section{Características y falencias del modelo de acreditación colombiano}

El proceso de acreditación de alta calidad colombiano se fundamenta en tres etapas de acuerdo con los lineamientos del Consejo Nacional de Acreditación (CNA): (1) autoevaluación, (2) evaluación externa, y (3) reconocimiento oficial. Este trabajo tiene un alcance que, por ahora, sólo abarca la primera etapa, dentro de la cual se deben analizar el cumplimiento en niveles de excelencia de diez factores que agrupan cuarenta características, las cuales, a su vez, deben ser analizadas dando respuesta a aspectos específicos que cada IES y cada programa pueden definir, tomando como base una batería sugerida por el cNA.

Las principales falencias del sistema de aseguramiento de la calidad se pueden sintetizar en tres (Blasco, 2011; Colombia. Ministerio de Educación Nacional, 2018; Gómez Campo \& Celis Giraldo, 2009):

1) En la búsqueda de resultados inmediatos es fácil caer en la afanosa costumbre, bien arraigada en algunos estudiantes, que privilegian la aprobación sobre el logro académico. Esta realidad se correlaciona con las enormes dificultades conceptuales sobre la naturaleza de la tarea y la ambigüedad del concepto de calidad que está inmerso en los lineamientos de acreditación.

2) Se privilegian las fortalezas sobre las debilidades; naturalmente, en el anhelo de obtener resultados positivos, la subjetividad de los autores de los informes de autoevaluación llega a ser sobredimensionada (Blasco, 2011).

3) Tienen poco impacto en el desarrollo de las funciones misionales de una IES. Esto es casi imposible de superar en los primeros estadios del proceso, puesto que la metodología y el rigor de los procesos de autoevaluación son mucho más ambiguos e inmaduros, respecto a los procesos de planeación estratégica que suelen ser una adaptación de lo que se utiliza exitosamente en el sector empresarial (Karathanos \& Karathanos, 2005). Pero luego de una maduración propia de los ejercicios de autoevaluación con la metodología del CNA, los resultados deberían ser de gran impacto, 
teniendo en cuenta que además el reconocimiento de acreditación de alta calidad es temporal, precisamente porque es necesario monitorear que se haya dado mejora continua y en especial, que sea significativa en las características de mayor importancia. De allí la necesidad de establecer una ponderación bien justificada.

En la actualidad, las IES se han reformulado, de acuerdo a un esquema mercantilista en el que el conocimiento está preponderantemente enfocado en la investigación aplicada para los artefactos industriales, que se monetizan a través de la extensión y el pago de las matrículas de estudiantes que ven en la educación una forma de inversión, con rentabilidades a futuro (Rama, 2009). Este escenario ha sido determinante para la definición de estructuras administrativas que se adaptan a una demanda de eficiencia para volverse más competitivas en el mercado. Pero la definición de esa arquitectura empresarial reviste una complejidad creciente en correlación con la evolución del número de matriculados. Esto se puede evidenciar en la expansión de los organigramas y la distribución de tareas cada vez más específicas en diferentes áreas de la organización (Perrucci \& Mannweiler, 1968).

Como se ilustrará a continuación, la noción compleja del concepto de calidad es esencial para una comprensión más completa y coherente con la realidad. Y al hacerlo, se desprende una amplitud de abordajes metodológicos e instrumentos para medir y gestionar.

\section{Multi-definición y complejidad del concepto de calidad en educación superior}

La calidad es un concepto complejo (Acosta Ochoa, 2015; Cabrera, 2005; Harvey \& Green, 1993; Larrauri, Espinosa, \& Robles, 2015). En parte esa complejidad tiene que ver con la multi-definición entre los actores de una comunidad universitaria, que en todo caso pueden tener consenso sobre la preponderancia de la calidad como compromiso ético al punto de incorporarlo en la cultura organizacional (Harvey \& Stensaker, 2008; Mavrogianni, 2018); pero no necesariamente hay una definición consensuada, al interior de una IES, del concepto de calidad.

La multi-definición se puede caracterizar en al menos dos dimensiones. De un lado, es razonable pensar que las expectativas como proceso y resultado pueden ser diferentes según los roles de una comunidad universitaria. Así, los estudiantes abogan por docentes de calidad, sin necesidad de autocriticarse; y viceversa, los docentes ven en la calidad de los estudiantes un 
elemento clave para juzgar el proceso educativo, sin necesidad de ahondar en un ejercicio autocrítico. Cada uno valora de manera distinta aspectos claves de la calidad. En el lenguaje de la acreditación, cada uno los pondera de forma diferente. De otro lado, en una segunda dimensión, la calidad adquiere una connotación muy diferente y ciertamente ilusoria cuando se analiza en fragmentos que describen una parte o el resultado del proceso educativo (De la Orden, 2009). Por ejemplo, algunas veces las IES se esfuerzan mucho para conseguir resultados notables en investigación, a través de la producción de los docentes con el fin de subir en la clasificación estandarizada (Hedrick, Henson, Krieg, \& Wassell, 2010; Iossifova, 2008); y no es evidente cuál es la influencia de ese logro en la calidad del proceso educativo como un todo, circunstancia que redunda en una multi- definición en la que hay calidad en aspectos puntuales, pero no en la globalidad. Así, se configura un problema que a la vez convoca a ponderar y establecer relaciones entre los elementos evaluados.

Usualmente en la literatura se da por sentado que la calidad de la educación superior está en función del desempeño y la percepción de los egresados, las relaciones pedagógicas, la productividad de los docentes y la visibilidad internacional (Gómez Campo \& Celis Giraldo, 2009). En específico, la noción de calidad está muy atada a la función de docencia (Albert et al., 2000; Grossman, Hammerness, \& McDonald, 2009; Healey, 2000; Loewenberg Ball \& Forzani, 2009; Nicholls, 2002). Esos referentes teóricos asumen implícitamente una definición de los aspectos determinantes de la calidad, que no está justificada más que por los antecedentes teóricos. Como alternativa, en este trabajo se estudian las interrelaciones que se tejen entre las partes (características según los lineamientos de acreditación) con que se evalúa la calidad, según los lineamientos de acreditación del CNA.

En toda organización, la información se construye y adquiere sentido en las interacciones entre las partes, y por lo tanto, es necesario comprender el fenómeno educativo como un sistema (Bertalanffy \& Almela, 1976) ;las implicaciones de adoptar este enfoque se visibilizan en la definición del concepto de calidad y su correspondiente medición (Dooley \& Van de Ven, 1999; Frank \& Fahrbach, 1999). En suma, esta concepción sistémica se inscribe en una concepción compleja de la calidad que se sustenta en los siguientes aspectos:

- Es un sistema auto-organizado que depende del contexto (Morin, 2009): de acuerdo a los postulados teóricos mencionados con antelación, complejidad no está en las posibles repuestas a múltiples preguntas que indagan sobre características aisladas, sino en la interrelación entre ellas. Pues bien, esas interconexiones emergen en el proceso educativo dándole sentido o razón de ser a cada una. Las definen en un contexto organizado en for- 
ma de Institución. Como producto de la gestión, emergen nuevos órdenes dentro de una institución que resignifican el papel de una característica o factor que las agrupa, pero siempre en función de las interacciones que se tienen en la globalidad del ejercicio.

- La autoorganización lleva implícita la indeterminación de las nuevas organizaciones que emergen como resultado de la gestión creativa. En ese devenir, es de vital importancia el contexto externo a la organización concebida como sistema abierto que se transforma en función de lo que recibe de otros sistemas como el mercado laboral, las políticas públicas, las crisis y las emergencias de nuevos paradigmas en la ciencia, las realidades y los momentos históricos de lo local y lo internacional.

- Es subjetiva: en la medida que la calidad tiene posibilidades válidas de multi-definición, como también de posibilidades de multi-valoración. De hecho, el asunto es parte del proceso de acreditación en la etapa de valoración externa por medio de pares académicos y consejeros que proveen decisiones finales. Lo que se encuentra allí, es que los evaluadores - académicos de diferentes disciplinas que están "prestados" al SNA - involucran su criterio personal basado en la experiencia en sus respectivas IES. También hay subjetividad en los actores de la IES que hacen su autoevaluación (Colombia. Ministerio de Educación Nacional, 2018).

- Es un concepto dialógico: la disertación sobre la calidad como principio rector de la función educadora da espacio a la posibilidad de ubicarla en el lugar del tercero incluido (Nicolescu, 1996); con esta lógica, un elemento como la calidad puede tener dos propiedades en principio contradictorias. Por ejemplo, la categoría "alta calidad" puede tenerse y no tenerse al mismo tiempo en una misma unidad de análisis, dígase un programa a de una IES $\beta$. Y no hay razón para creer que no puede ser así. Esto se corresponde con la multi-definición del concepto en función del contexto ilustrado antes, que genera posiciones en conflicto a la hora de hacer juicios de valor. Una imperfección cuya eliminación al parecer es imposible o al menos indeseable, dado que su existencia conlleva a un diálogo que necesita procedimientos de validez y rigor articulados a la argumentación para extraer resultados valiosos que mejoren la calidad de la educación superior.

\section{Uso de redes en los procesos de autoevaluación}

La estructura organizacional de una IES suele ser una jerarquía que reparte funciones misionales a través de ramas bien establecidas en un organigrama. Esta administración es lineal y simplificada en tanto da cuenta de las relaciones de poder y en alguna medida de flujo de información a través de las diferentes áreas de la organización. Sin embargo, a través del aprendizaje institucional y con buena gestión de procesos se pueden evidenciar las relaciones entre dependencias como producto de experiencias de trabajo que 
planeadas o no necesitan de un buen flujo de información para que operen bien. Más que una documentación de los pasos que se deben llevar para completar una tarea, un sistema de procesos describe una organización como una red de comunicación (Bolton \& Dewatripont, 1994) Conocer el funcionamiento de esa red, la fuerza y la naturaleza de sus conexiones, así como su evolución en el tiempo, es fundamental para una gestión exitosa enfocada a conseguir logros a través de un liderazgo que es capaz de conocer el equipo de trabajo"(Flap, Bulder, \& Völker, 1998.)

Más allá del tratamiento de las relaciones al interior de las organizaciones, el uso de redes se ha expandido a sistemas macro en los que interactúan varias personas o instituciones, y desde ese enfoque se descubren propiedades del conjunto que antes no eran visibles en cada una de las partes (Borgatti \& Foster, 2003). En el contexto específico de la calidad, el uso de redes es de gran utilidad para establecer una ponderación en un enfoque general que no necesita asumir, a priori, la medición del logro educativo como lo más importante, tal como sucede en los modelos de acreditación estadounidenses (Hussain, Mak, \& Addas, 2016). Este problema reviste gran importancia en la gestión de la calidad en tanto ayuda a contener el problema de las fortalezas sobredimensionadas de las que se comentó al principio, porque puede suceder que se encuentren en aspectos de menor importancia. Más aún, el proceso de ponderación es clave porque ayuda a establecer un referente que permita priorizar la administración de recursos de todo tipo en función de la mejora continua.

Sin alguna razón clara a la vista, el modelo de acreditación de alta calidad colombiano eliminó la ponderación como instrumento obligatorio, dejándolo a voluntad de cada IES. Una de las razones para eliminar este requisito es que el ordenamiento debe tener una justificación. Si se tiene en cuenta que el modelo de acreditación tiene 40 características para evaluar, en una primera aproximación reduccionista, la justificación pasa por la necesidad de explicar las razones por las cuales una característica es más importante que otra. En total, el número de comparaciones a justificar es el conteo que resulta de aplicar esta fórmula general: $N=\sum_{k=1}^{n}(n-k)$. Donde $N=$ es el número de comparaciones; y $n=$ número de características evaluadas. Con 40, que es el mínimo contemplado en el modelo colombiano, las comparaciones serían 480. Con la restricción, que todas ellas deben cumplir con el principio de transitividad para lograr un ordenamiento racional (Regenwetter, Dana, \& Davis-Stober, 2011)y ésta a su vez es más importante que una C, entonces A debe ser más importante que $C$. Un procedimiento de esta naturaleza es casi imposible de construir en el contexto de un proceso de autoevaluación con 
fines de acreditación. En microeconomía, a un problema inabordable por el raciocinio humano, como éste que se acaba de presentar, se le asigna una restricción denominada "racionalidad limitada", y por ende, teniendo solución no se espera que cualquier persona pueda encontrarla.

Por lo anterior, es más racional y consistente abandonar la concepción reduccionista, para ahondar en una más posible fundamentada en la concepción sistémica, haciendo uso de los extraordinarios avances de la teoría de redes. Una red expresa las relaciones entre características (llamados vértices) que se relacionan de manera fuerte o débil. En una o doble vía.

Una buena introducción a la teoría de redes se encuentra en Steen, (2010). Lo que es importante subrayar es la capacidad de este instrumento para revelar al menos cuatro rasgos de complejidad del concepto de calidad en un ejercicio de autoevaluación (Ver Tabla 1. Propiedades de complejidad que son visibles en una red.)

Tabla 1. Propiedades de complejidad que son visibles en una red.

\begin{tabular}{ll}
\hline Propiedades de complejidad & Características de una red \\
\hline Sistémica & $\begin{array}{l}\text { Una red establece relaciones entre las partes y demuestra que hay } \\
\text { propiedades importantes inmersas en esas relaciones. }\end{array}$ \\
\hline Holo gramática & $\begin{array}{l}\text { Las redes densas, recogen mucha información sobre todo el } \\
\text { sistema en pocos nodos muy bien conectados. }\end{array}$ \\
\hline Recursividad & $\begin{array}{l}\text { Las redes pueden ser no - dirigidas; es decir pueden establecer } \\
\text { relaciones de doble vía entre dos elementos conectados. }\end{array}$ \\
\hline Autoorganización & $\begin{array}{l}\text { La estructura de la red se puede "descubrir" después de que la } \\
\text { IES lleva muchos años funcionando; en vez de definirla. E irá } \\
\text { migrando hacia estructuras más o menos impredecibles. }\end{array}$ \\
\hline
\end{tabular}

Fuente: elaboración propia

Con el uso de una red, la ponderación se logra no a través de la difícil discusión acerca de qué es más importante en el proceso educativo comparando todos los pares posibles, sino a través de la identificación de las relaciones fuertes o débiles entre las características. En un escenario ideal, estas relaciones no requieren de una justificación única para cada relación, puesto que pueden extraerse de la información de los procesos y procedimientos académicos en IES con sistemas de gestión por procesos automatizados. Aunque este asunto es bien extenso y daría para un trabajo independiente, de lo que se trata es de establecer métricas de interacción en cada uno de los procedi- 
mientos académicos claves y luego homologarlas en la estructura de características del modelo de acreditación. Sin embargo, para este trabajo se exploró un camino distinto. En el contexto del proceso de autoevaluación con fines de acreditación de un programa, se convocó a un grupo de expertos para definir el relacionamiento entre características que constituye una red.

\section{Recolección de datos}

El ejercicio aplicado de este trabajo es resultado del proceso de acreditación de alta calidad del programa de Ingeniería en Telecomunicaciones de la Institución Universitaria Politécnico Grancolombiano. Para definir una ponderación de las cuarenta características, se convocó a cinco profesores de planta del programa - con más de dos años de antigüedad - a un taller con metodología cualitativa de discusión (Alcalá, Villaverde, Benito, \& García, 2017). En la primera parte del taller, se les presentó la justificación del ejercicio y el enfoque de redes para establecer una ponderación, usando conceptos similares a los esbozados en este artículo entorno a la noción sistémica y de complejidad del concepto de calidad y excelencia.

En la segunda parte del taller se les pidió establecer relaciones ponderadas entre las características, con el razonamiento inmerso en esta oración: ¿Qué tanto requiere la característica A de la característica B, para alcanzar niveles de excelencia? En este punto es importante mencionar que la pregunta orientadora permite pasar de la mirada lineal planteada anteriormente en la cual se enfatizaba en cuál es la característica más importante, a la mirada sistémica de red, enfocando en las relaciones entre las características. Para completar la tarea se les entregó, con antelación al desarrollo del taller, un documento con la definición de cada característica y los aspectos que la componen, de acuerdo con el modelo del CNA.

Con las instrucciones ilustradas, los profesores diligenciaron una matriz de relaciones mutuas, entre cada característica y todas las demás, a través de una hoja de cálculo editable en línea. El nivel de relación tiene una escala que toma valores de 0,1,2 (donde 0 es relación casi nula, 1 es relación débil y 2 es relación fuerte).

Las relaciones definidas por los cinco profesores fueron promediadas para obtener una matriz final que se modela como una red. 


\section{Metodología}

\section{Modelación en red}

Una vez se tienen definidas las relaciones a través de una matriz cuadrada, se pueden usar al menos dos métricas para establecer una ponderación en función de las características sistémicas: 1) el grado de entrada y salida, que asigna importancia a un vértice (característica) en función de la cantidad de relaciones, teniendo en cuenta que algunas son más fuertes que otras de acuerdo con la escala que se definió en el taller. 2) El grado expresado en el "eigen vector" de la matriz de las relaciones. Se trata de una variación de la primera medida en el que se premia, en nivel de importancia (ponderación), el hecho de tener relaciones con otros vértices fuertemente relacionados (Steen, 2010).

Detrás del cálculo del nivel de importancia, o ponderación en el lenguaje de la acreditación de alta calidad, hay un cálculo numérico que sería muy dispendioso de realizar sin la ayuda de una herramienta computacional. Por lo tanto, para este caso, se utilizó el software estadístico R y el paquete especializado en redes i-graph (G. Csardi \& Nepusz, 2006; M. G. Csardi, 2013) que dispone de funciones suficientes para la visualización y el cálculo de la importancia de los vértices de acuerdo con las dos métricas seleccionadas.

\section{Identificación de comunidades}

La estructura de Factores-Características-Aspectos establece una agrupación a priori, que se fundamenta en la similitud de lo que se reúne a nivel de característica o factor. En la detección de comunidades, se conserva al máximo esa estructura y se identifican nuevas agrupaciones a través de la identificación de subredes densas, por medio de un algoritmo computacional que puede identificar subredes pequeñas o grandes, denominadas "comunidades".

En este trabajo se utiliza el algoritmo "fast greedy" que detecta subredes densas grandes (Clauset, Newman, \& Moore, 2004). A diferencia de algoritmos orientados a identificar subredes densas pequeñas, que pueden ser de interés en otros contextos, la identificación de comunidades de factores y características grandes conlleva a una interpretación coherente y útil para el análisis de la transmisión de acciones de mejoramiento en todo el proceso educativo. 


\section{Resultados}

Las dos métricas de importancia de las características expresadas como nodos en la red arrojan resultados similares. En el Gráfico 1 se presenta la red de características usando la ponderación "eigen vector". En esta visualización, las características se representan mediante nodos de la red. Tanto la distancia del centro como el tamaño de cada nodo (característica) está en función del nivel de ponderación. Los colores agrupan las características dentro de los factores de acreditación definidos según los lineamientos del cNA. Complementariamente, en la Tabla 2 se presenta, en una escala porcentual, la ponderación de características para las dos métricas. El histograma del Gráfico 2 refleja una distribución ancha de las ponderaciones, lo que permite determinar agrupaciones bien definidas, con ponderaciones muy similares y a la vez diferencias significativas entre las características más importantes.

Gráfico 1. Red de características ponderadas

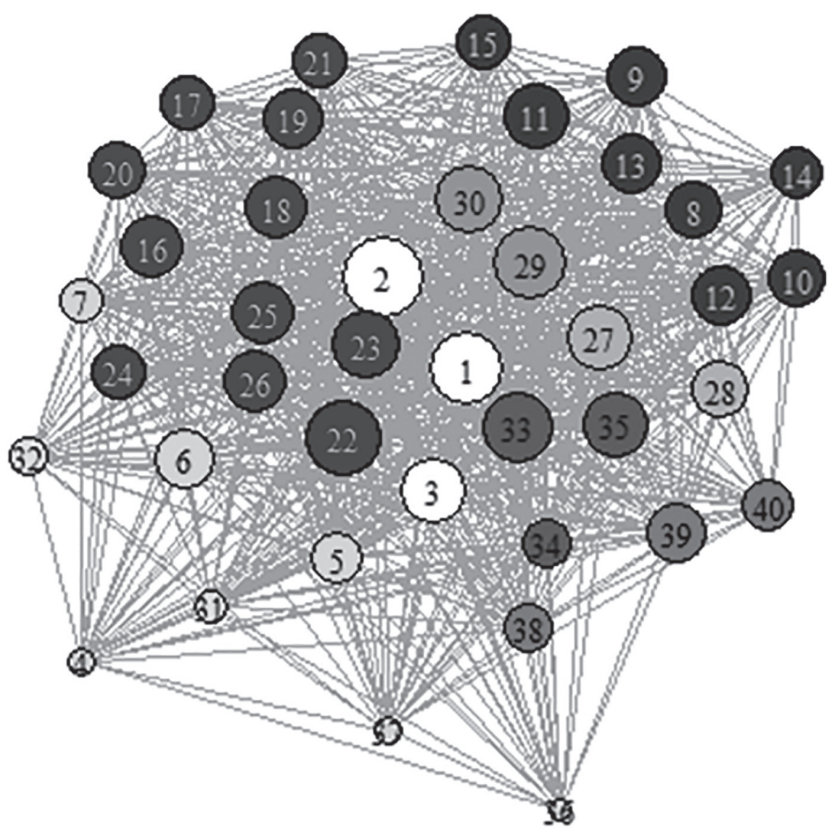

Fuente: elaboración propia 
Gráfico 2. Histograma de ponderación de características

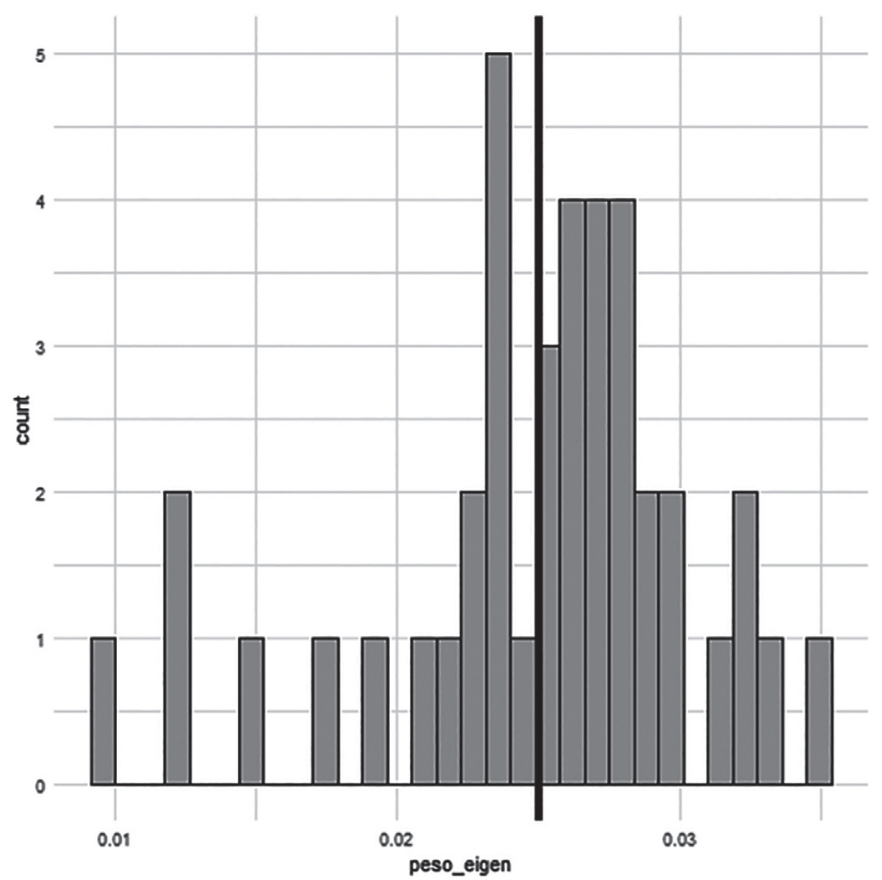

Fuente: elaboración propia.

Tabla 2. Ponderación de características y pertenencia a comunidades

\begin{tabular}{|c|c|c|c|c|c|c|}
\hline No. & Factor & $\begin{array}{c}\text { ID } \\
\text { Característica }\end{array}$ & Característica & peso_eigen & peso_out_in & Comunidad \\
\hline 1 & $\begin{array}{l}\text { Misión, proyecto } \\
\text { institucional y de } \\
\text { programa }\end{array}$ & 2 & $\begin{array}{l}\text { Proyecto Educativo } \\
\text { del Programa }\end{array}$ & $3.52 \%$ & $3.59 \%$ & 2 \\
\hline 4 & $\begin{array}{l}\text { Procesos acadé- } \\
\text { micos }\end{array}$ & 22 & $\begin{array}{l}\text { Evaluación y } \\
\text { autorregulación del } \\
\text { programa }\end{array}$ & $3.32 \%$ & $3.41 \%$ & 2 \\
\hline 1 & $\begin{array}{l}\text { Misión, proyecto } \\
\text { institucional y de } \\
\text { programa }\end{array}$ & 1 & $\begin{array}{l}\text { Misión, Visión y Pro- } \\
\text { yecto Institucional }\end{array}$ & $3.26 \%$ & $3.37 \%$ & 3 \\
\hline 6 & $\begin{array}{l}\text { Investigación, } \\
\text { innovación y } \\
\text { creación artística } \\
\text { y cultural }\end{array}$ & 29 & $\begin{array}{l}\text { Formación para } \\
\text { la investigación, } \\
\text { la innovación y la } \\
\text { creación artística y } \\
\text { cultural }\end{array}$ & $3.24 \%$ & $3.19 \%$ & 1 \\
\hline 8 & $\begin{array}{l}\text { Organización, } \\
\text { administración y } \\
\text { gestión }\end{array}$ & 33 & $\begin{array}{l}\text { Organización, admi- } \\
\text { nistración y gestión } \\
\text { del programa. }\end{array}$ & $3.12 \%$ & $3.19 \%$ & 3 \\
\hline 6 & $\begin{array}{l}\text { Investigación, } \\
\text { innovación y } \\
\text { creación artística } \\
\text { y cultural }\end{array}$ & 30 & $\begin{array}{l}\text { Compromiso con la } \\
\text { investigación y la } \\
\text { creación artística y } \\
\text { cultural }\end{array}$ & $3.01 \%$ & $2.95 \%$ & 1 \\
\hline
\end{tabular}


Frederick A. Mendoza L., Mary N. Ortegon C. / Revista de la Educación Superior 48 (192) (2019) 1-21

\begin{tabular}{|c|c|c|c|c|c|c|}
\hline 4 & $\begin{array}{l}\text { Procesos acadé- } \\
\text { micos }\end{array}$ & 23 & $\begin{array}{l}\text { Extensión o proyec- } \\
\text { ción social }\end{array}$ & $2.93 \%$ & $2.93 \%$ & 2 \\
\hline 8 & $\begin{array}{l}\text { Organización, } \\
\text { administración y } \\
\text { gestión }\end{array}$ & 35 & $\begin{array}{l}\text { Dirección del pro- } \\
\text { grama }\end{array}$ & $2.87 \%$ & $2.92 \%$ & 3 \\
\hline 5 & $\begin{array}{l}\text { Visibilidad } \\
\text { nacional e inter- } \\
\text { nacional }\end{array}$ & 27 & $\begin{array}{l}\text { Inserción del progra- } \\
\text { ma en contextos aca- } \\
\text { démicos nacionales e } \\
\text { internacionales }\end{array}$ & $2.86 \%$ & $2.88 \%$ & 1 \\
\hline 3 & Profesores & 11 & Desarrollo profesoral & $2.82 \%$ & $2.76 \%$ & 1 \\
\hline 1 & $\begin{array}{l}\text { Misión, proyecto } \\
\text { institucional y de } \\
\text { programa }\end{array}$ & 3 & $\begin{array}{l}\text { Relevancia acadé- } \\
\text { mica y pertinencia } \\
\text { social del programa }\end{array}$ & $2.81 \%$ & $2.83 \%$ & 2 \\
\hline 4 & $\begin{array}{l}\text { Procesos acadé- } \\
\text { micos }\end{array}$ & 18 & Interdisciplinariedad & $2.80 \%$ & $2.76 \%$ & 2 \\
\hline 4 & $\begin{array}{l}\text { Procesos acadé- } \\
\text { micos }\end{array}$ & 26 & $\begin{array}{l}\text { Recursos de apoyo } \\
\text { docente }\end{array}$ & $2.78 \%$ & $2.77 \%$ & 2 \\
\hline 4 & $\begin{array}{l}\text { Procesos acadé- } \\
\text { micos }\end{array}$ & 16 & $\begin{array}{l}\text { Integralidad del } \\
\text { currículo }\end{array}$ & $2.72 \%$ & $2.69 \%$ & 2 \\
\hline 4 & $\begin{array}{l}\text { Procesos acadé- } \\
\text { micos }\end{array}$ & 25 & $\begin{array}{l}\text { Recursos informáti- } \\
\text { cos y de comuni- } \\
\text { cación }\end{array}$ & $2.69 \%$ & $2.69 \%$ & 2 \\
\hline 2 & Estudiantes & 6 & $\begin{array}{l}\text { Participación en } \\
\text { actividades de for- } \\
\text { mación integral }\end{array}$ & $2.68 \%$ & $2.69 \%$ & 2 \\
\hline 3 & Profesores & 12 & $\begin{array}{l}\text { Estímulos a la do- } \\
\text { cencia, investigación, } \\
\text { creación artística y } \\
\text { cultural, extensión } \\
\text { o proyección social } \\
\text { y a la cooperación } \\
\text { internacional }\end{array}$ & $2.68 \%$ & $2.64 \%$ & 1 \\
\hline 3 & Profesores & 13 & $\begin{array}{l}\text { Producción, perti- } \\
\text { nencia, utilización e } \\
\text { impacto de material } \\
\text { docente }\end{array}$ & $2.65 \%$ & $2.61 \%$ & 1 \\
\hline 10 & $\begin{array}{l}\text { Recursos físicos } \\
\text { y financeros }\end{array}$ & 39 & $\begin{array}{l}\text { Presupuesto del } \\
\text { programa }\end{array}$ & $2.63 \%$ & $2.64 \%$ & 3 \\
\hline 4 & $\begin{array}{l}\text { Procesos acadé- } \\
\text { micos }\end{array}$ & 19 & $\begin{array}{l}\text { Estrategias de ense- } \\
\text { ñanza y aprendizaje }\end{array}$ & $2.62 \%$ & $2.59 \%$ & 2 \\
\hline 3 & Profesores & 9 & Estatuto profesoral & $2.61 \%$ & $2.55 \%$ & 1 \\
\hline 3 & Profesores & 10 & $\begin{array}{l}\text { Número, dedicación, } \\
\text { nivel de formación } \\
\text { y experiencia de los } \\
\text { profesores }\end{array}$ & $2.56 \%$ & $2.50 \%$ & 1 \\
\hline 3 & Profesores & 8 & $\begin{array}{l}\text { Selección, vincula- } \\
\text { ción y permanencia } \\
\text { de profesores }\end{array}$ & $2.52 \%$ & $2.47 \%$ & 1 \\
\hline 5 & $\begin{array}{l}\text { Visibilidad } \\
\text { nacional e inter- } \\
\text { nacional }\end{array}$ & 28 & $\begin{array}{l}\text { Relaciones externas } \\
\text { de profesores y } \\
\text { estudiantes. }\end{array}$ & $2.52 \%$ & $2.50 \%$ & 1 \\
\hline 4 & $\begin{array}{l}\text { Procesos acadé- } \\
\text { micos }\end{array}$ & 20 & $\begin{array}{l}\text { Sistema de evalua- } \\
\text { ción de estudiantes }\end{array}$ & $2.47 \%$ & $2.45 \%$ & 2 \\
\hline 4 & $\begin{array}{l}\text { Procesos acadé- } \\
\text { micos }\end{array}$ & 24 & $\begin{array}{l}\text { Recursos bibliográ- } \\
\text { ficos }\end{array}$ & $2.40 \%$ & $2.36 \%$ & 2 \\
\hline
\end{tabular}

Continúa.. 


\begin{tabular}{|c|c|c|c|c|c|c|}
\hline 3 & Profesores & 15 & $\begin{array}{l}\text { Evaluación de } \\
\text { profesores }\end{array}$ & $2.36 \%$ & $2.27 \%$ & 1 \\
\hline 4 & $\begin{array}{l}\text { Procesos acadé- } \\
\text { micos }\end{array}$ & 17 & $\begin{array}{l}\text { Flexibilidad del } \\
\text { currículo }\end{array}$ & $2.36 \%$ & $2.26 \%$ & 2 \\
\hline 4 & $\begin{array}{l}\text { Procesos acadé- } \\
\text { micos }\end{array}$ & 21 & $\begin{array}{l}\text { Trabajos de los } \\
\text { estudiantes }\end{array}$ & $2.36 \%$ & $2.34 \%$ & 2 \\
\hline 3 & Profesores & 14 & $\begin{array}{l}\text { Remuneración por } \\
\text { méritos }\end{array}$ & $2.34 \%$ & $2.30 \%$ & 1 \\
\hline 2 & Estudiantes & 5 & $\begin{array}{l}\text { Estudiantes admi- } \\
\text { tidos y capacidad } \\
\text { institucional }\end{array}$ & $2.28 \%$ & $2.41 \%$ & 3 \\
\hline 10 & $\begin{array}{l}\text { Recursos físicos } \\
\text { y financieros }\end{array}$ & 40 & $\begin{array}{l}\text { Administración de } \\
\text { recursos }\end{array}$ & $2.26 \%$ & $2.26 \%$ & 3 \\
\hline 8 & $\begin{array}{l}\text { Organización, } \\
\text { administración y } \\
\text { gestión }\end{array}$ & 34 & $\begin{array}{l}\text { Sistemas de comuni- } \\
\text { cación e información. }\end{array}$ & $2.17 \%$ & $2.22 \%$ & 3 \\
\hline 10 & $\begin{array}{l}\text { Recursos físicos } \\
\text { y financieros }\end{array}$ & 38 & Recursos Físicos & $2.14 \%$ & $2.15 \%$ & 3 \\
\hline 2 & Estudiantes & 7 & $\begin{array}{l}\text { Reglamentos estu- } \\
\text { diantil y académico }\end{array}$ & $1.94 \%$ & $1.96 \%$ & 2 \\
\hline 7 & $\begin{array}{l}\text { Bienestar } \\
\text { institucional }\end{array}$ & 32 & $\begin{array}{l}\text { Permanencia y reten- } \\
\text { ción estudiantil }\end{array}$ & $1.77 \%$ & $1.84 \%$ & 3 \\
\hline 7 & $\begin{array}{l}\text { Bienestar } \\
\text { institucional }\end{array}$ & 31 & $\begin{array}{l}\text { Políticas, programas } \\
\text { y servicios de bienes- } \\
\text { tar universitario }\end{array}$ & $1.49 \%$ & $1.56 \%$ & 3 \\
\hline 9 & $\begin{array}{l}\text { Impacto de los } \\
\text { egresados en el } \\
\text { medio }\end{array}$ & 37 & $\begin{array}{l}\text { Impacto de los egre- } \\
\text { sados en el medio } \\
\text { social y académico }\end{array}$ & $1.24 \%$ & $1.27 \%$ & 2 \\
\hline 2 & Estudiantes & 4 & $\begin{array}{l}\text { Mecanismos de } \\
\text { selección e ingreso }\end{array}$ & $1.21 \%$ & $1.26 \%$ & 3 \\
\hline 9 & $\begin{array}{l}\text { Impacto de los } \\
\text { egresados en el } \\
\text { medio }\end{array}$ & 36 & $\begin{array}{l}\text { Seguimiento a los } \\
\text { egresados }\end{array}$ & $0.99 \%$ & $1.00 \%$ & 2 \\
\hline
\end{tabular}

Fuente: elaboración propia

El ejercicio de ponderación expresa una concepción de la calidad que se fundamenta en los propósitos de formación delineados en el proyecto educativo del programa (característica con mayor ponderación) y el proyecto educativo institucional (Tercera característica con mayor ponderación). Otorga una importancia alta a los procesos de evaluación y autorregulación de programa (segunda característica con mayor ponderación), que dan cuenta de una cultura de excelencia académica evidenciable a través de los resultados de la organización-administración y gestión del programa (quinta característica en ponderación).

Las otras características que pertenecen al grupo de las diez con más alta ponderación expresan con claridad la importancia de las tres funciones sustantivas de la educación superior: 1) docencia atada al desarrollo profesoral, 2) el compromiso con la investigación y 3) la proyección social. Estas características son impactadas positivamente por la inmersión del programa en 
las redes internacionales que pueden establecer formas de cooperación o de referenciación de experiencias entorno a la excelencia académica.

\section{Definición de comunidades}

El modelo de acreditación cNA establece un agrupamiento de cada una de las cuarenta características en diez factores. El ejercicio de relacionamiento que se modela en red no exigió a los integrantes del taller que tuviesen en cuenta ese agrupamiento, en el sentido de asignar niveles de relacionamiento similares entre las características de un mismo factor y todas las demás. Sin embargo, era de esperarse que al modelar la red en función del nivel de importancia "eigen", hubiese similitud entre los niveles de importancia. Esto se refleja en las claras sectorizaciones que exhiben los colores del Gráfico 1, debido a que claramente las características de un mismo factor tratan sobre aspectos muy parecidos.

La definición de comunidades es de gran importancia, en la medida que su identificación justifica la aplicación de acciones de mejoramiento focalizadas en un grupo de características con mayor ponderación que en todo caso tienen impacto sobre otras. En ese sentido el modelo de gestión se enfoca en prever los mecanismos de transmisión de las acciones de mejoramiento en una red que establece un impacto a priori sobre un número definido de características y aspectos que se ven influenciados, en diferente escala. Este impacto se entiende como positivo en la medida que la metodología fue clara en señalar al núcleo profesoral que diligenció el instrumento, la relación de complementariedad y aporte a la excelencia en los aspectos de cada característica, derivado de los impactos positivos del grupo con el que se conecta.

La detección de comunidades por el método "fast greedy" amplía la agrupación a priori por factores según el lineamiento CNA. Los resultados son consistentes dado que, para ocho de los diez factores, la agrupación calculada asigna las características de un mismo factor, en una misma comunidad. (Ver Tabla 3).

Tabla 3. Número de características de cada factor en cada comunidad.

\begin{tabular}{llll}
\hline \multirow{2}{*}{ Factor } & \multicolumn{3}{c}{$\mathbf{N}^{\circ}$ Características en cada comunidad } \\
\cline { 2 - 4 } & 1 & 2 & 3 \\
\hline 1 & & 2 & 1 \\
\hline 2 & & 2 & 2 \\
\hline 3 & 8 & & \\
\hline
\end{tabular}




\begin{tabular}{lll}
\hline 4 & & 11 \\
\hline 5 & 2 & \\
\hline 6 & 2 & \\
\hline 7 & & 2 \\
\hline 8 & & 3 \\
\hline 9 & & \\
\hline 10 & & 3 \\
\hline
\end{tabular}

Fuente: elaboración propia

El primer factor ubica dos características en la comunidad 2 y una en la comunidad 3. Para simplificar, se asume que este factor pertenece a la comunidad 2. El segundo factor ubica dos características en la comunidad 2 y dos en la comunidad 3. Ante este empate, se asume que pertenece a dos comunidades $(2 \mathrm{y}$ 3). Los demás factores ubican todas sus características en una sola comunidad (Ver Tabla 3). Tomando ese resultado, en el Gráfico 3 se presenta la red de factores agrupados por las comunidades a las que pertenecen sus características.

\section{Gráfico 3. Red de factores en las tres comunidades}
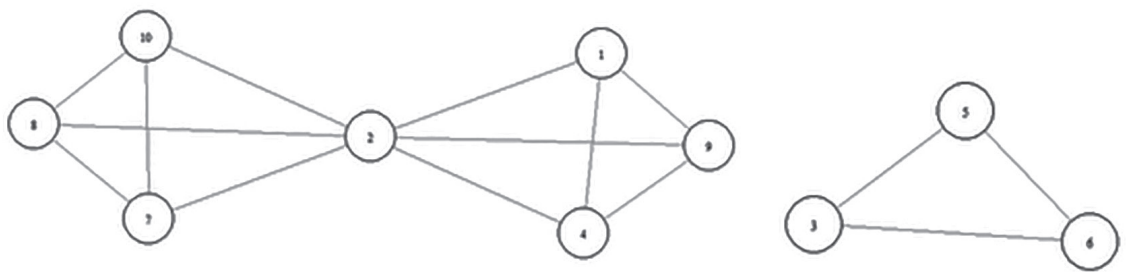

Fuente: elaboración propia

En este punto, es importante aclarar que este método de agrupación, derivado de la aplicación de un algoritmo sobre la red, identifica comunidades que resaltan niveles altos de conexión (subredes densas) entre características. Este agrupamiento se correlaciona, como era de esperarse, con la de factores, definida con base en la similitud de las temáticas abordadas en las características. Sin embargo, como se ilustró en la sección de resultados y más específicamente en la representación del Gráfico 1, no existen características o factores completamente aislados.

Una agrupación en tres comunidades establece entonces un primer nivel de análisis de transmisión general que es más alto y simple. La red sin la agrupación de comunidades permite verificar la transmisión a niveles más detallados. 


\section{Análisis de los resultados de agrupación en comunidades}

Comunidad 1: agrupa los factores de profesores (factor 3), visibilidad nacional e internacional (factor 5) e investigación (factor 6). Resalta por medio de esta relación el liderazgo del rol docente en los procesos de internacionalización e investigación, que es de hecho una forma de desarrollar la "internacionalización en casa". Este concepto está abordado en los aspectos que desarrollan las características de ese factor y está orientado a ampliar la función de la internacionalización, más allá de la movilidad entrante y saliente de estudiantes profesores. Así se ha ido afianzando el concepto de internacionalización del curriculum de la investigación, la docencia y la proyección social, bien sea por la consolidación de redes internacionales de trabajo que cooperan en línea, o incluso la incorporación de referentes teóricos o buenas prácticas de IEs en el mundo a la gestión de las funciones sustantivas (Arce González, 2015; Beelen \& Jones, 2015; Jon, 2013; Soria \& Troisi, 2014).

Comunidad 2: agrupa los factores de misión, proyecto institucional y de programa (factor 1), procesos académicos (factor 4), estudiantes (factor 2: a esta comunidad sólo pertenecen las características de participación en actividades de formación integral y reglamento estudiantil), e impacto de los egresados en el medio (factor 9). Esta agrupación es de gran interés en la medida que articula el proceso educativo desde la raíz que se expresa en los proyectos educativos y de programa, con el desarrollo curricular caracterizado en los procesos académicos haciendo acento en la participación de los estudiantes en la formación integral y la claridad en las reglas de juego que se deben expresar en los reglamentos. Finalmente, vincula los aspectos observables del resultado del proceso educativo reflejados en el desempeño de los egresados.

Comunidad 3: agrupa los factores de organización, administración y gestión (factor 8), recursos físicos y financieros (factor 10), bienestar institucional (factor 7) y estudiantes (factor 2: a esta comunidad sólo pertenecen las características de mecanismos de selección e ingreso y capacidad intelectual). Esta agrupación agrupa claramente las características y aspectos asociados al soporte administrativo y financiero, que le dan soporte a todo el proceso educativo en una IES.

Es muy llamativo que articule el factor de bienestar universitario, en la media que no es evidente la relación entre las características de ese factor y las demás involucradas en esta comunidad. Es claro que al tratarse de un ejercicio que parte de la apreciación subjetiva de un grupo de profesores en torno al relacionamiento, este resultado revela una visión que le asigna un lugar administrativo a un factor que es más reconocido como influyente en la formación integral (Becerra Heraud, 2013). 


\section{Conclusiones}

Este trabajo estuvo motivado en la identificación de tres falencias señaladas en la literatura crítica sobre el sistema de aseguramiento de la calidad colombiano, que están relacionadas con la ausencia de una metodología adecuada para evaluar la calidad de los programas de educación superior. El mayor reto consiste en desarrollar definiciones, instrumentos y valoraciones que den cuenta de la complejidad que adquiere el concepto en el contexto educativo, tarea de gran valor y que requiere un desarrollo investigativo extenso.

Se ha propuesto un instrumento puntual con base en la modelación de un sistema que dé cuenta de las interacciones entre las partes (características), asunto en el que la teoría de redes (Newman, 2010) tiene mucho que aportar. Este tipo de modelos permiten recolectar mucho mejor la información detallada de las interacciones a través de la definición de matrices de relación (Hurtado, 2005) que usualmente se aplican en la teoría administrativa de la decisión (Rodríguez-Bárcenas \& López-Huertas, 2013) y ayudan a descubrir propiedades complejas de las que no se da cuenta en los enfoques reduccionistas típicamente utilizados. En particular, este trabajo ilustra la manera como este instrumento permite establecer una ponderación bien justificada y aporta a la construcción de una metodología más rigurosa.

La detección de comunidades a través de una partición en subredes establece, en un primer nivel, un instrumento sencillo para evaluar el impacto de las acciones de mejoramiento a través de la transmisión entre los factores y características conectados. En un nivel más detallado, la red completa que se dibuja en función de los niveles de ponderación también es útil para evaluar esa transmisión. Sin embargo, dado que los datos del ejercicio especifico que se expuso están sujetos a la propuesta de relacionamiento de un grupo de expertos, y no parte de una base de datos extraída de un sistema de información, la validez de los mecanismos de transmisión debe hacerse en la práctica y puede dar insumos para perfeccionar la matriz de relaciones inicial.

No hay ninguna restricción a la vista respecto a la reproducibilidad de este modelo de ponderación en el contexto de otros lineamientos para la evaluación de la calidad distintos al colombiano. En cualquier caso, así como en este trabajo se parte de una definición de las relaciones entre cuarenta características, en otros referentes de evaluación, el mismo relacionamiento se puede definir de acuerdo con la taxonomía de elementos a evaluar, que pueden estar en términos de factores o dimensiones o incluso indicadores.

Para investigaciones posteriores, la modelación de redes se fundamentará en las métricas de los procesos académicos, en las IES que los tienen sistematizados a través de una herramienta estandarizada para tal fin. Esto ayudará a 
pasar de la simulación a un ejercicio más realista de las relaciones que existen y su magnitud. También permitirá enlazar la gestión desde la planeación estratégica, en donde comúnmente se analiza el desempeño de los procesos académicos, con los resultados ponderados del proceso de autoevaluación que concibe las propiedades complejas del concepto de calidad del que pretende dar cuenta.

\section{Referencias}

Acosta Ochoa, A. (2015). La FIMPES y la mejora de la calidad de instituciones privadas: Un estudio acerca del concepto de calidad y de los procesos de acreditación en tres universidades particulares. Revista de La Educación Superior, 44(175), 169-175.

Albert, B., Kenny, S., Booth, W., Glaser, M., Glassick, C., \& Ikenberry, S. (2000). Reinventing undergraduate education: A blueprint for America's research universities. The Boyer Commission on Educating Undergraduates in the Research University.

Alcalá, D. H., Villaverde, V. A., Benito, V. D., \& García, V. A. (2017). Análisis de la importancia de los criterios de evaluación y el reconocimiento académico docente universitario como indicadores de la calidad educativa en España. Revista de La Educación Superior, 46(181), 75-87.

Arce González, A. M. (2015). Internacionalización y Educación Superior. Revista de La Educación Superior, 44(176), 165-170.

Becerra Heraud, S. (2013). Universidades saludables: una apuesta a una formación integral del estudiante. Revista de Psicología (PUCP), 31(2), 287-314.

Beelen, J., \& Jones, E. (2015). Redefining internationalization at home. In The European higher education area (pp. 59-72). Springer.

Bertalanffy, L. von, \& Almela, J. (1976). Teoría general de los sistemas: fundamentos, desarrollo, aplicaciones. Fondo de Cultura Económica,.

Blasco, C. M. (2011). Calidad, evaluación y acreditación en la educación superior: a propósito de un proyecto de reforma en Colombia. Pensamiento Jurídico, (31), 155-185. Retrieved from http://www.bdigital.unal.edu.co/7385/

Bolton, P., \& Dewatripont, M. (1994). The Firm as a Communication Network. The Quarterly Journal of Economics, 109(4), 809-839. https://doi. org/10.2307/2118349

Borgatti, S. P., \& Foster, P. C. (2003). The network paradigm in organizational research: A review and typology. Journal of Management, 29(6), 991-1013. https:/ / doi.org/10.1016/S0149-2063(03)00087-4

Cabrera, V. A. (2005). El concepto calidad en la educación universitaria: clave para el logro de la competitividad institucional. Revista Iberoamericana de Educación, 36(12), 1-7.

Clauset, A., Newman, M. E. J., \& Moore, C. (2004). Finding community structure in very large networks. Physical Review E, 70(6), 66111. 
Colombia. Ministerio de Educación Nacional. (2018). Referentes de calidad: una propouesta para la evolución del sistema de aseguramiento de la calidad. Bogotá.

Csardi, G., \& Nepusz, T. (2006). The igraph software package for complex network research. InterJournal, Complex Systems, 1695(5), 1-9.

Csardi, M. G. (2013). Package 'igraph.' Last Accessed, 3(09), 2013.

De la Orden, A. (2009). Evaluación y calidad: análisis de un modelo. Estudios Sobre Educacion, (16), 17-36.

Dooley, K. J., \& Van de Ven, A. H. (1999). Explaining complex organizational dynamics. Organization Science, 10(3), 358-372.

Flap, H., Bulder, B., \& Völker, B. (1998). Intra-organizational Networks and Performance: A Review. Computational \& Mathematical Organization Theory, 4(2), 109-147. https:/ / doi.org/10.1023/ A:1009675906926

Frank, K. A., \& Fahrbach, K. (1999). Organization culture as a complex system: Balance and information in models of influence and selection. Organization Science, 10(3), 253-277.

Gómez Campo, V. M., \& Celis Giraldo, J. E. (2009). Sistema de aseguramiento de la calidad de la educación superior: consideraciones sobre la acreditación en Colombia.

Grossman, P., Hammerness, K., \& McDonald, M. (2009). Redefining teaching, reimagining teacher education. Teachers and Teaching: Theory and Practice, 15(2), 273-289. https:// doi.org/10.1080/13540600902875340

Harvey, L., \& Green, D. (1993). Defining Quality. Assessment E Evaluation in Higher Education, 18(1), 9-34. https:/ / doi.org/10.1080/0260293930180102

Harvey, L., \& Stensaker, B. (2008). Quality culture: Understandings, boundaries and linkages. European Journal of Education, 43(4), 427-442. https://doi. org/10.1111/j.1465-3435.2008.00367.x

Healey, M. (2000). Developing the scholarship of teaching in higher education: a discipline-based approach. Higher Education Research \& Development, 19(2), 169-189.

Hedrick, D. W., Henson, S. E., Krieg, J. M., \& Wassell, C. S. (2010). The Effects of AACSB Accreditation on Faculty Salaries and Productivity. Journal of Education for Business, 85(5), 284-291. https:/ / doi.org/10.1080/08832320903449543

Hurtado, L. H. (2005). Un modelo de ponderación para la autoevaluación de un programa academico. Scientia Et Technica, (29), 69-73.

Hussain, W., Mak, F. K., \& Addas, M. F. (2016). Engineering program evaluations based on automated measurement of performance indicators data classified into cognitive, affective, and psychomotor learning domains of the revised Bloom's taxonomy. In ASEE Annual Conference and Exposition, Conference Proceedings (Vol. 2016-June).

Iossifova, A. (2008). Research Productivity, Teaching Relevance, and AACSB Accreditation. Quality Management Journal, 15(4), 46-56. https:/ / doi.org/10.108 0/10686967.2008.11918204

Jon, J.-E. (2013). Realizing internationalization at home in Korean higher education: Promoting domestic students' interaction with international students and intercultural competence. Journal of Studies in International Education, $17(4), 455-470$. 
Karathanos, D., \& Karathanos, P. (2005). Applying the balanced scorecard to education. Journal of Education for Business, 80(4), 222-230.

Larrauri, J. O., Espinosa, E. M., \& Robles, M. I. P. (2015). La diversidad semántica y el carácter político de las nociones de calidad en la Educación Superior de México. Revista de La Educación Superior, 44(173), 85-102.

Loewenberg Ball, D., \& Forzani, F. M. (2009). The work of teaching and the challenge for teacher education. Journal of Teacher Education, 60(5), 497-511.

Mavrogianni, R. (2018). Quality culture as a condition for the quality assurance of an educational institution. Academia (Greece), (11), 83-102.

Morin, E. (2009). Introducción al pensamiento complejo (1st ed.). Buenos Aires: Gedisa.

Newman, M. (2010). Networks: an introduction. Oxford university press.

Nicholls, G. (2002). Developing teaching and learning in higher education. Routledge.

Nicolescu, B. (1996). La transdisciplinariedad: manifiesto. Multiversidad Mundo Real Edgar Morin, AC.

Perrucci, R., \& Mannweiler, R. A. (1968). Organization size, complexity, and administrative succession in higher education. Sociological Quarterly, 9(3), 343-355.

Rama, C. (2009). La Universidad Latinoamericana en la encrucijada de sus tendencias. Universidad Nacional Autónoma de Honduras UNAH.

Regenwetter, M., Dana, J., \& Davis-Stober, C. P. (2011). Transitivity of Preferences. Psychological Review, 118(1), 42-56. https:/ / doi.org/10.1037/a0021150

Rodríguez-Bárcenas, G., \& López-Huertas, M. J. (2013). Saaty's analytic hierarchies method for knowledge organization in decision making. Journal of the Association for Information Science and Technology, 64(7), 1454-1467.

Soria, K. M., \& Troisi, J. (2014). Internationalization at home alternatives to study abroad: Implications for students' development of global, international, and intercultural competencies. Journal of Studies in International Education, 18(3), 261-280.

Steen, M. Van. (2010). Graph theory and complex networks: An introduction. (Vol. 144). Amsterdam: Marteen Van Steen. 
